\title{
Progressive lung disease in a malt-worker
}

\author{
ME ELLIS, JAR FRIEND \\ From the Department of Thoracic Medicine, City Hospital, Aberdeen
}

We describe a malt-worker whose initial symptoms suggested extrinsic allergic alveolitis. The ensuing cavitating lung disease, Aspergillus fumigatus infection with mycetomata, vigorous immune response, and granulomatous liver disease are unusual features.

\section{Case report}

A 32-year-old man started work for a distillery company, driving a hopper lorry. Over seven years he was exposed almost daily to the dust of barley, malt, and draff when he entered the enclosed hopper to sweep it out. During the last three years he noticed wheezing within minutes of sweeping, followed about four hours later by transitory sweating, cough, and breathlessness. Despite relinquishing this work, he experienced progressive breathlessness on exertion. He presented at hospital aged 42 years, thin, with marked finger clubbing and a few fine basal lung crackles on auscultation.

Chest radiography showed reticulo-nodular shadowing in both lungs, and upper and mid zone fibrosis with bullae (fig 1). Pulmonary function testing indicated a restrictive defect and reduction of transfer factor. The blood count was normal, ESR was $62 \mathrm{~mm}$ in the first hour. There was no blood or sputum eosinophilia. Sputum culture grew Aspergillus fumigatus repeatedly. Tuberculin testing (10 TU) was negative. Tubercle bacilli were not isolated from the sputum. Aspergillus fumigatus precipitin tests were strongly positive. Prick tests to Aspergillus fumigatus and a variety of allergens were negative, with no late reactions. Aspergillus clavatus has never been identified either in the sputum or serologically. A Kveim test was negative, as were precipitin tests for farmer's lung and bird fancier's lung. Autoimmune screening including lung antibody was negative. Bronchoscopy demonstrated right upper and middle lobe bronchiectasis. Plasma immunoglobulin $\mathbf{G}$ and $A$ levels were high (643 and $266 \mathrm{mg} / \mathrm{l}$ respectively). Liver function tests were often temporarily abnormal. He was known to drink alcohol sporadically. Liver biopsy showed marked granulomatous change with epithelioid and giant cells, but no evidence of an alcoholic cirrhosis. Staining and culture for tubercle bacilli, Brucella, and fungi were negative. A complement consumption assay detected circulating immune complexes in the serum, but no evidence of immune complex deposition in the liver was forthcoming using specific rabbit anti-Aspergillus serum.

Address for reprint requests: Dr ME Ellis, Department of Infectious Diseases, Monsall Hospital, Manchester.

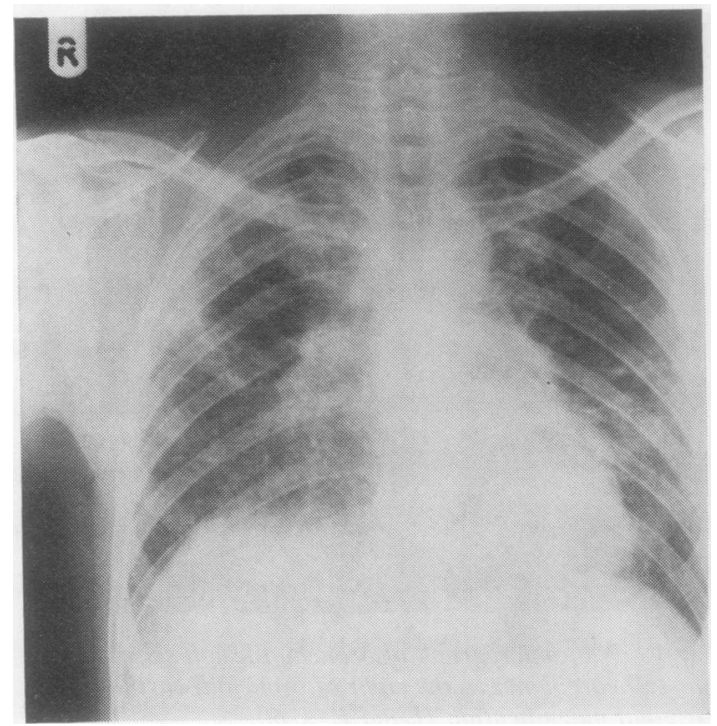

Fig 1 Initial chest radiograph showing bilateral reticulo-nodular shadowing.

Chest radiographs have shown progressive fibrosis in upper and mid zones with mycetoma formation (fig 2). Lung biopsy was not thought justified in view of his poor respiratory function.

Despite intensive courses of prednisolone and pre- $\frac{\text { 을 }}{\partial}$ cautionary anti-tuberculous chemotherapy, his condition $D$ deteriorated. A four-week course of intravenous econazole resulted in improved well-being, a temporary $\mathrm{N}$ reduction of Aspergillus sputum colony counts, and $\sigma$ improved carbon monoxide transfer factor.

\section{Discussion}

Aspergillus fumigatus has been associated with asthma, extrinsic allergic bronchioalveolitis, aspergillomata, $\mathbb{\mathscr { C }}$ invasive aspergillosis, and allergic bronchopulmonary? aspergillosis. Asthma and allergic bronchopulmonary aspergillosis can be discounted in this patient as there were no asthmatic features, fleeting pulmonary shadows, $\stackrel{\mathbb{Q}}{\stackrel{Q}{Q}}$ blood or sputum eosinophilia, or positive prick tests.

The initial symptoms were those of an extrinsice allergic bronchioalveolitis consistent with malt-worker's lung. ${ }^{1}$ In a survey of 711 Scottish malt-workers, Aspergil-응 


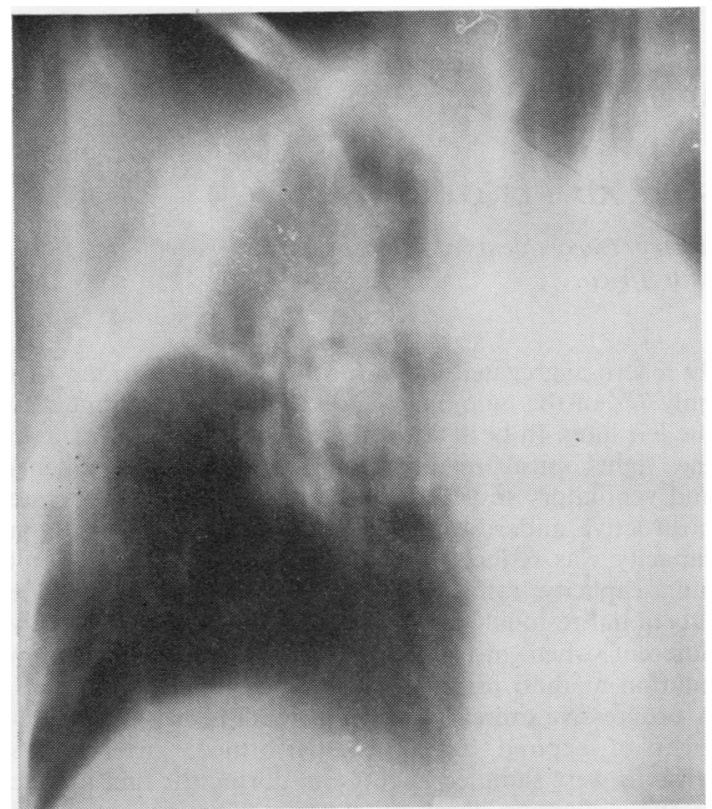

Fig 2 Tomogram of right lung showing upper zone fibrosis and aspergilloma.

lus clavatus was documented as the causative fungus. ${ }^{1}$ In our patient, however, there has been no microbiological or serological evidence for Aspergillus clavatus, the findings suggesting a pathogenic, possibly primary, role for Aspergillus fumigatus. This organism has only once been incriminated in malt-worker's lung, ${ }^{2}$ despite a spore size $(3 \mu \mathrm{m})$ suitable for alveolar deposition.

Alternatively, colonisation by Aspergillus fumigatus, of lung previously damaged by Aspergillus clavatus, might have occurred producing mycetomata. Aspergillus fumigatus colonisation has been reported secondary to histoplasmosis, ${ }^{3}$ and occurs in healed tuberculous cavities.

Aspergillomata do not usually affect mortality or morbidity adversely, but they can occasionally cause ill health and recurrent haemoptysis. Progressive lung destruction in aspergillomata is unusual, but has occurred in this patient. Although the morphology is not that of purely invasive type of Aspergillus, the extreme immunological reaction (circulating immune complexes, extremely high immunoglobulins and multiple precipitin lines) may be the result of Aspergillus antigens leaching out of the mycetomata and reaching immunologically active cells.

Pulmonary granuloma formation occurs in extrinsic allergic bronchioalveolitis but the aetiology is not clear. ${ }^{4}$ Whereas the association of cryptogenic fibrosing alveolitis and liver disease is known, liver granulomata have not been described in extrinsic allergic bronchioalveolitis. Their significance in this patient is unclear as we could not confirm the presence of immune complexes within them, despite the presence of circulating immune complexes. However they may well be a tissue manifestation of extreme hypersensitivity to Aspergillus fumigatus; one explanation for the "absence" of immune complexes in the granulomata maybe the activation of the alternative pathway of complement. ${ }^{5}$ It is less likely that the granulomata could represent disseminated aspergillosis. This rarely occurs except in severe immunodeficient states.

With currently available antifungal agents it is unlikely that Aspergillus fumigatus can be eradicated from the mycetomata in this patient, although econazole may have reduced the antigen load temporarily. The disease is far too widespread for surgical intervention. Attempts to suppress the immunological reaction with corticosteroids have not prevented deterioration. Plasmapheresis would be another possible approach, of uncertain benefit.

We are grateful to Dr DT Kay, Dr LGR Milne, Dr T Reid, and Dr CHW Horne for their help.

\section{References}

${ }^{1}$ Grant IBW, Blackadder ES, Greenberg M, Blyth W. Extrinsic allergic alveolitis in Scottish maltworkers. Br Med J 1976;1:490-3.

2 Vallery-Radot LP, Giroud P. Sporomycose des pelleteurs de grains. Bull Soc Mèd Hôp Paris 1928:52:1632-45.

${ }^{3}$ Procknow JJ, Loewen DF. Pulmonary aspergillosis with cavitation secondary to histoplasmosis. Am Rev Respir Dis 1960;82:101-11.

4 Turner-Warwick M. Extrinsic allergic bronchioalveolitis. In: Immunology of the lung. London: Edward Arnold, 1978:182-3.

5 Edwards JH. A quantitative study on the activation of the alternative pathway of complement by mouldy hay dust and thermophilic actinomycetes. Clin Allergy 1976;6:19-25. 